proteins from different manufacturers should be re-evaluated. Because the divergent V3 loop of the LAV-1/IIIB isolate may yield atypical neutralization profiles, comparative trials with more typical isolates might be of greater value. The politics of env are real, but let us hope that the ability of scientists to change is not inversely proportional to that of our opponent, HIV.

John P. Moore and Robin A. Weiss are at the Chester Beatty Laboratories, Institute of Cancer Research, 237 Fulham Road, London SW3 6JB, UK.

\title{
Bringing molecules to order
}

\section{Richard Friend}

METHODS that allow controlled growth of ordered films of molecular or polymeric materials are much sought after for a range of potential applications. With this in mind, Wittmann and Smith take a fresh look, on page 414 of this issue ${ }^{1}$, at experiments performed some years ago on the nature of friction processes when poly(tetrafluoroethylene) is drawn across a smooth surface $^{2}$. The friction produces highly ordered and oriented thin layers of poly(tetrafluoroethylene) which Wittmann and Smith now find can be used as versatile and effective substrates for the growth of ordered films of molecular materials. This provides an important new method for preparing a wide range of structures and will be of particular interest to those working on the development of molecular materials for electronics, or molecular electronics.

Poly(tetrafluoroethylene) is well known to show very low coefficients of friction against a range of surfaces, and is widely used for this property. Pooley and Tabor $^{3}$ studied the processes that occur as this polymer is drawn across a smooth surface, such as glass, and found that a thin (typically $5 \mathrm{~nm}$ ) film of the polymer is formed on the surface as polymer chains that are in contact with the surface are drawn out of the bulk. Slipping occurs not at the interface, but between the bulk of the polymer and this thin surface layer which is highly oriented. The low friction arises as aligned chains slide freely past one another. It is this thin layer, transferred to the underlying surface, that is used by Wittmann and Smith as a substrate on which oriented films can be grown.

For molecular materials which have a strong tendency to order, such as liquid crystals, oriented substrates are widely used to bring about alignment of molecular layers in a well-defined direction on a substrate (rubbed polyimide layers are used in most liquid crystal displays). The alignment of a nematic liquid crystal on thin, oriented poly(tetrafluoroethylene) layers produced frictionally has previously been used to provide an image of the underlying order in the polymer layer under a polarizing microscope ${ }^{4}$. Wittmann and Smith have shown that a much wider range of materials can be oriented in this way, including many, such as polyaniline, that do not show a strong tendency to order.

Control of order in polymers is central to the control of both mechanical and electronic properties. A well-known example is the range of mechanical properties which can be obtained in polyethylene of varying molecular weight and draw ratio. As a lowmolecular-weight material, its elastic moduli and strength are not impressive, but highly oriented ultrahigh-molecularweight polyethylene shows a modulus and tensile strength close to the theoretical strength determined by the intrachain covalent carbon-carbon bond strength $^{5}$.

\section{Conductivlty}

Turning to electronic properties, conjugated polymers such as polyacetylene conduct metallically when oxidized or reduced, and levels of conductivity have risen to become comparable to those of good metals (copper's is 600,000 siemens (S) per centimetre) since interest first arose in this in the late 1970s. What has been less clear is the way in which conductivity is controlled by order in the polymer. But recent work on drawn fibres of various conjugated polymers reveals a linear relationship between electrical conductivity on the one hand and tensile strength or Young's modulus on the other, rising with increasing draw ratio. Values of $30,000 \mathrm{~S} \mathrm{~cm}^{-1}$ and 0.9 or 50 gigapascals, respectively, are reached for polyacetylene ${ }^{6}$ and $1,200 \mathrm{~S} \mathrm{~cm}^{-1}$ and 0.7 or 35 gigapascals for poly $(2,5$ dimethoxy phenylene vinylene) (ref. 7). This correlation between modulus and conductivity provides an important clue to the processes that limit the electronic mobility. The high levels of both that are reached for high draw ratios point to many areas of application.

Molecular materials are harder to process than polymers, but there is interest in electronic properties ranging from nonlinear optical properties, such as second-harmonic generation, to metallic and superconducting behaviour. Growth, layer by layer, of films by the Langmuir-Blodgett technique is well developed, but this is constrained by the requirement that the material to be deposited forms a Langmuir film on the surface of the transfer medium (usually water). Most materials put down in this way are designed with long aliphatic tails which cause the molecules to stand on end on the water surface. It can be undesirable, however, to have a large volume fraction of the film that is electronically inactive, and there are now some rigid-rod polymers (phthalocyanato-polysiloxanes ${ }^{8}$ and polyglutamates ${ }^{9}$ ) which have been developed to lie flat on the water surface. When transferred to form the LangmuirBlodgett film these align with the direction of draw of the substrate, and provide films of high quality.

Electronic mobilities in organic semiconductors are strongly influenced by the level of order in the material, and these have usually been found to be extremely low, of the order of $10^{-4} \mathrm{~cm}^{2}$ $\mathrm{V}^{-1} \mathrm{~s}^{-1}$ at room temperature, and characteristic of transport by thermallyactivated motion from one molecular site to the next. Garnier et al. ${ }^{10,11}$ have shown that it is possible to use sexithiophene (an oligomer of the conjugated polymer polythiophene) as the active layer in a field effect transistor and to achieve mobilities as high as 0.4 $\mathrm{cm}^{2} \mathrm{~V}^{-1} \mathrm{~s}^{-1}$. These molecular films were deposited by the simple means of sublimation in vacuo, but it now seems that these structures may be unexpectedly well ordered. Recent X-ray measurements on similar films of thiophene oligomers show a highly-ordered, crystalline packing of molecules giving good intermolecular contact ${ }^{12}$. The technique now developed by Wittmann and Smith provides a particularly versatile method that will allow fabrication of ordered molecular films drawn from a wider range of materials than previously possible.

Richard Friend is at the Cavendish Laboratory. Madingley Road, Cambridge CBЗ OHE, UK.

1. Wittmann. J. C. \& Smith, P. Nature 352, 414-417 (1991)

2. Bowden, F. P. \& Tabor, D. The Friction and Lubrication of Solids, Part $/ l$ (Clarendon, Oxford, 1964).

3. Pooley, C. M. \& Tabor, D. Proc. R. Soc. A329, 251-274 (1972)

4. Tanaka, K. \& Miyata, T. Wear 41, 383-398 (1977)

5. Smith, P. \& Lemstra P. J. J. Mater. Sci. 15, 505 (1980)

6. Cao. Y., Smith, P. \& Heeger, A. J. Polymer 32, 1210 1218 (1991).

7. Tokito, S., Smith, P. \& Heeger, A. J. Polymer 32 464-470 (1991).

8. Caseri, W., Sauer, T. \& Wegner, G. Makromol. Chem Rapid Commun. 9, 651-657 (1988)

9. Duda, G. et al. Thin Solid Films 159, 221-230 (1988)

10. Garnier, F., Horovitz, G., Peng, X. \& Fichou, D. Adv Mater. 2, 592-594 (1990)

11. Bloor, D. Nature 349, 738-740 (1991).

12. Akimichi, H., Waragai, K., Hotta, S., Kano, H. \& Sakai, H. Appli. Phys. Lett. 58, 1500-1502 (1991) 